\title{
The spin dynamics of molecular magnets beyond Kubo's linear response theory
}

\author{
C.A. Dartora* \\ Electrical Engineering Department, Federal University of Parana (UFPR) \\ G.G. Cabrera ${ }^{\dagger}$ \\ Instituto de Física 'Gleb Wataghin', Universidade Estadual de Campinas (UNICAMP), 13.083-970 São Paulo, Brazil \\ K.Z. Nobrega \\ Electrical Engineering Department, CEFET-MA
}

(Received on 28 May, 2009)

\begin{abstract}
The description of quantum dynamics of nanomagnets is a central issue in most applications proposed for those systems. In this paper, we put forward a modified perturbation approach to study the spin dynamics of a molecular magnet in the presence of time-dependent magnetic fields. The non-perturbed Hamiltonian $H_{0}$, which defines the interaction picture, may be time-dependent proviso it can be diagonalized at all times by the same basis of states. We probe the method using a simple model Hamiltonian, that contains the important anisotropy terms relevant for $\mathrm{Fe}_{8}$ molecular clusters, and solve as an example the case with the smallest non trivial spin value $(S=1)$. Our modified perturbation approach converges rapidly to the exact solution, goes beyond the Kubo linear response theory, and is well defined even at resonance. Temperature effects in the spin dynamics are taken into account in the context of the density matrix.
\end{abstract}

Keywords: nanomagnets, spin dynamics, Kubo response theory, perturbation theory

\section{INTRODUCTION}

Molecular nanomagnets with high-spin and high anisotropy exhibit extraordinary effects, namely the pronounced magnetic hysteresis and the quantization of the magnetic moment[1, 2]. Promising applications of such magnets for current and future technologies include information storage, construction of nanomagnetic Maser-like devices[3], and quantum computation. The name SingleMolecule Magnets (SMM) has been coined to mean that individual molecules in a crystal act as individual magnets and the interaction between two distinct molecules are negligible, in general. They can be prepared in longlived excited quantum states by simply applying a magnetic field[4], and exhibit a stepwise magnetic hysteresis in a timedependent magnetic field. Quantum relaxation of spin states at low temperatures is very sluggish, and has been intensively studied within the framework of the Landau-ZenerStückelberg(LZS) theory[5-8]. At low temperature, quantum tunneling is of primary importance for the magnetic relaxation process. In the adiabatic regime, the LZS theory predicts an exponentially small tunneling probability between states which are near a level crossing. However, a reliable account of the general dynamics, beyond the LZS approach, is highly desirable to include interesting physical phenomena with time-dependent fields[8]. In addition, real-time dynamics is of prime importance to perform quantum computation, to understand the time evolution and decoherence effects of quantum bits [9]. Quantum mechanically exact, if possible, as well as accurate approximate solutions are welcomed within this framework[10]. They are superior to numerical computations, since they allow to grasp qualita-

*Electronic address: cadartora@eletrica.ufpr.br

${ }^{\dagger}$ Electronic address: cabrera@ifi.unicamp.br tively the basic physics involved in a given problem. Timedependent Hamiltonians constitute a distinguished class of problems, usually solved through the time-dependent perturbation theory in the context of the interaction picture (or Dirac version of quantum mechanics)[11]. The basic assumption in the usual interaction picture, is the possibility to separate a given Hamiltonian $\mathcal{H}$, into a non-perturbed timeindependent part $\mathcal{H}_{0}$ and a time-dependent perturbation $V(t)$, which is assumed to be 'small'. We note that representative examples of such problems, precisely include spin dynamics of molecular magnets in the presence of time-dependent fields[1]. The usual low-order time-dependent perturbation theory has several shortcomings: to lowest order, the theory is limited to short intervals of time; and, at resonances, the theory breaks down no matter how small the perturbation.

In this paper we will apply a modified version of the interaction picture, already used in a previous manuscript[3], to study the spin dynamics of a molecular magnet under the influence of a time-dependent magnetic field. We show that out approach allows one to go beyond the usual Kubo linear response theory. Temperature effects are taken into account through the density matrix formalism.

The content of this paper can be described as follows: in the next Section we briefly discuss the mathematical framework of the modified interaction picture. In Section III the simplest form of a SMM Hamiltonian is presented. In Section IV we consider a more realistic approach and temperature is introduced. Finally, in the last Section a few remarks and conclusions are added.

\section{THEORETICAL FRAMEWORK}

We start with a mathematical description the modified version of the interaction picture, in which we split the Hamil- 
tonian $\mathcal{H}$ into two time-dependent parts $\mathcal{H}_{0}$ and $V(t)$ :

$$
\mathcal{H}=\mathcal{H}_{0}(t)+V(t) .
$$

The non-perturbed Hamiltonian $\mathcal{H}_{0}$ is time dependent and conditioned to be diagonalized at all times by the same basis of states in the Hilbert space, i.e.,

$$
\left[\mathcal{H}_{0}(t), \mathcal{H}_{0}\left(t^{\prime}\right)\right]=0, \forall\left(t, t^{\prime}\right) .
$$

This term includes contributions from time dependent fields which cannot be considered as small. By contrast, the timedependent perturbation $V(t)$, in general not diagonal in the basis of $\mathcal{H}_{0}$, i.e.,

$$
\left[\mathcal{H}_{0}(t), V(t)\right] \neq 0, \forall t
$$

but assumed to give s̀mall' contributions. The Schrödinger equation can be solved within the scheme of this modified interaction picture assuming the unitary transformation below

$$
U_{0}=\exp \left(-\frac{i}{\hbar} \int_{0}^{t} d t^{\prime} \mathcal{H}_{0}\left(t^{\prime}\right)\right)
$$

which transforms states and operators as follows:

$$
\begin{aligned}
|\psi\rangle_{I} & =U_{0}^{\dagger}|\psi\rangle, \\
\Omega_{I} & =U_{0}^{\dagger} \Omega U_{0},
\end{aligned}
$$

where the subscript $I$ stands for 'interaction picture', and kets and operators without any subscript are understood to be in the Shrödinger picture. An observable $O_{I}$ obeys a Heisenberg-like equation of motion with $\mathcal{H}_{0}(t)$

$$
\frac{d O_{I}}{d t}=\frac{1}{i \hbar}\left[O_{I}, \mathcal{H}_{0}\right]
$$

while the wave function $\left|\psi_{I}\right\rangle$ and the density matrix $\rho_{I}$ evolve in time according to Schrödinger-like equations with $\mathcal{H}_{\mathcal{F}}$ :

$$
\begin{aligned}
\mathcal{H}_{I}|\psi\rangle_{I} & =i \hbar \frac{\partial}{\partial t}|\psi\rangle_{I}, \\
\frac{\partial \rho_{I}}{\partial t} & =\frac{i}{\hbar}\left[\rho_{I}, \mathcal{H}_{I}\right],
\end{aligned}
$$

where $\mathcal{H}_{I}$ is defined by the expression below

$$
\mathcal{H}_{I}=U_{0}^{\dagger} V(t) U_{0}=\exp \left(\frac{i}{\hbar} \int_{0}^{t} d t^{\prime} \mathcal{H}_{0}\left(t^{\prime}\right)\right) V(t) \exp \left(-\frac{i}{\hbar} \int_{0}^{t} d t^{\prime} \mathcal{H}_{0}\left(t^{\prime}\right)\right)
$$

The temporal evolution of a given initial ket-state $\left|\Psi_{0}\right\rangle$ will be given by the unitary evolution operator $U_{I}$ in this modified interaction picture. The latter can be written in the form of a Dyson series[11]:

$$
\begin{aligned}
U_{I}(t, 0)= & 1-\frac{i}{\hbar} \int_{0}^{t} d t_{1} \mathcal{H}_{I}\left(t_{1}\right)+ \\
& +\left(\frac{-i}{\hbar}\right)^{2} \int_{0}^{t} d t_{1} \mathcal{H}_{I}\left(t_{1}\right) \int_{0}^{t_{1}} d t_{2} \mathcal{H}_{I}\left(t_{2}\right)+\ldots,
\end{aligned}
$$

with $|\Psi(t)\rangle_{I}=U_{I}(t, 0)\left|\Psi_{0}\right\rangle$. Following, in the next Section we discuss the usual Hamiltonian used to describe a molecular magnet.

\section{THE MOLECULAR MAGNET HAMILTONIAN}

One of the Hamiltonians used to model the spin system of molecular magnets, like the $\mathrm{Fe}_{8}$ molecular cluster [12-14], is given by :

$$
\mathcal{H}=-D S_{z}^{2}+E\left(S_{+}^{2}+S_{-}^{2}\right)-\mu \mathbf{S} \cdot \mathbf{B}(t),
$$

where $D$ is the uniaxial anisotropy constant, $E$ the anisotropy constant in the transverse plane, which may be small, and $\left(S_{z}, S_{ \pm}\right)$are the spin operators with units such that $\hbar=1$ $\left(S_{ \pm}=S_{x} \pm i S_{y}\right)$. The last term in (8) gives the coupling of the magnetic moment with a time dependent magnetic field $\mathbf{B}(t)$. If we could set $E=0$, in the absence of an external field $\mathbf{B}$, the ground state would be doubly degenerate, corresponding to the states with maximum parallel $(m=S)$ and anti-parallel $(m=-S)$ projections of the magnetic moment along the quantization axis. The action of $E$ admixes these states with other states of the spectrum, in the sequence $(|S\rangle,|S-2\rangle,|S-4\rangle, \ldots|-S+4\rangle,|-S+2\rangle,|-S\rangle)$, with a small coupling between $|-S\rangle$ and $|S\rangle$, the larger the value of $S$ the weaker the coupling. For large anisotropy, i.e. $E / D<<1$, the spin up $(m>0)$ and the spin down $(m<0)$ orientations of the molecular magnetic moment are separated by an effective energy barrier, which is responsible for the slow magnetic relaxation. At low temperatures, the sluggish relaxation effects are due to tunneling through the barrier.

For the sake of simplicity we consider the simplest nontrivial case of spin 1 molecules $(S=1)$, under the influence of a time-varying magnetic field with component along the quantization axis, taken to be along the $z$-direction. The operator $S_{z}$ is diagonal in the basis of states $\{|+1\rangle,|0\rangle,|-1\rangle\}$,

$$
S_{z}=\left(\begin{array}{ccc}
1 & 0 & 0 \\
0 & 0 & 0 \\
0 & 0 & -1
\end{array}\right)
$$

and for times $t<0$, i.e., before the applied magnetic field $\mathbf{B}(t)$ is turned on, we have the following Hamiltonian:

$$
\mathcal{H}_{<}=-D S_{z}^{2}+E\left(S_{+}^{2}+S_{-}^{2}\right) .
$$

We denote by $(|s\rangle,|0\rangle,|a\rangle)$ the basis which diagonalize $\mathcal{H}_{<}$, where $|0\rangle=|m=0\rangle$, and $(|s\rangle,|a\rangle)$ correspond to the tunnel- 
ing states:

$$
\begin{aligned}
& |s\rangle=\frac{1}{\sqrt{2}}(|+1\rangle+|-1\rangle), \\
& |a\rangle=\frac{1}{\sqrt{2}}(|+1\rangle-|-1\rangle) .
\end{aligned}
$$

Calling $\Delta=2 E$ the tunneling splitting, the corresponding eigenvalues of the energy are $E_{s}=-D+\Delta, E_{0}=0$, and $E_{a}=$ $-D-\Delta$. Notice that in this basis, ordered as $(|s\rangle,|0\rangle,|a\rangle)$, the $S_{z}$ operator is non-diagonal. In the next Section we will discuss the influence of temperature on the spin dynamics of a $S=1$ molecular magnet.

\section{SPIN DYNAMICS BEYOND KUBO'S LINEAR RESPONSE THEORY}

In the following, we introduce temperature and study the evolution of the density matrix $\rho$, when the system is perturbed by an oscillatory magnetic field whose polarization is near the z-axis:

$$
\begin{aligned}
\mathcal{H}_{<} & =-D S_{z}^{2}+E\left(S_{+}^{2}+S_{-}^{2}\right), t<0, \\
\mathcal{H} & =\mathcal{H}_{<}-\mu B_{0}(t) S_{z}-\frac{1}{\sqrt{2}} \mu\left(b(t) S_{+}+b^{*}(t) S_{-}\right), t \geq 0 \\
\rho_{0} & =\frac{e^{-\beta \mathcal{H}_{<}}}{\operatorname{Tr}\left(e^{\left.-\beta \mathcal{H}_{<}\right)}\right.}
\end{aligned}
$$

being $\beta=1 /\left(k_{B} T\right)$, and $\left|B_{0}(t)\right|>|b(t)|$. For $S=1$, the density matrix can be written in closed form as follows:

$$
\rho_{0}=\left(\begin{array}{ccc}
\gamma & 0 & \eta \\
0 & (1-2 \gamma) & 0 \\
\eta & 0 & \gamma
\end{array}\right)
$$

where we are using the basis of $S_{z}$. Quantities in (15) are given by:

$$
\begin{aligned}
& \gamma=\frac{e^{\beta D} \cosh (2 \beta E)}{2 e^{\beta D} \cosh (2 \beta E)+1}, \\
& \eta=-\frac{e^{\beta D} \sinh (2 \beta E)}{2 e^{\beta D} \cosh (2 \beta E)+1}
\end{aligned}
$$

For times $t \geq 0$ we break the Hamiltonian as follows:

$$
\begin{aligned}
\mathcal{H}_{0} & =-D S_{z}^{2}-\mu B_{0}(t) S_{z} \\
V(t) & =E\left(S_{+}^{2}+S_{-}^{2}\right)-\frac{1}{\sqrt{2}} \mu\left(b(t) S_{+}+b^{*}(t) S_{-}\right) .
\end{aligned}
$$

Writing $U_{0}=\exp \left(-i \int_{0}^{t} d t^{\prime} \mathcal{H}_{0}\left(t^{\prime}\right)\right)$, the perturbation in the interaction picture $U_{I}=1-i \int_{0}^{t} d t^{\prime} U_{0}^{\dagger} V\left(t^{\prime}\right) U_{0}$ is given by

$$
U_{I}=1-i\left(\begin{array}{ccc}
0 & I & J \\
I^{*} & 0 & K \\
J^{*} & K^{*} & 0
\end{array}\right)
$$

to first order approximation, with the definitions

$$
\begin{aligned}
& I=-\mu \int_{0}^{t} d t^{\prime} b(t) \exp \left(-i D t^{\prime}\right) \exp \left(-i \mu \int_{0}^{t^{\prime}} d t^{\prime \prime} B_{0}\left(t^{\prime \prime}\right)\right) \\
& J=2 E \int_{0}^{t} d t^{\prime} \exp \left(-2 i \mu \int_{0}^{t^{\prime}} d t^{\prime \prime} B_{0}\left(t^{\prime \prime}\right)\right) \\
& K=-\mu \int_{0}^{t} d t^{\prime} b(t) \exp \left(i D t^{\prime}\right) \exp \left(-i \mu \int_{0}^{t^{\prime}} d t^{\prime \prime} B_{0}\left(t^{\prime \prime}\right)\right)
\end{aligned}
$$

Observe in (21), (22), and (23), that corrections are linear in $(E, b(t))$, while contributions from $B_{0}(t)$ are considered to all orders.

The exact quantum Liouville equation (5) yields a set of coupled differential equations for the 5 independent components $\rho_{m n}$ of the matrix $\rho$, which are solved exactly by numerical methods. Concerning our approximate scheme, the evolution of the density matrix is evaluated by the expression:

$$
\rho(t)=U_{I} \rho_{0} U_{I}^{\dagger}
$$

and since the approximate operator $U_{I}$ from (20) is not unitary, we renormalize the density matrix at all times. The average of the spin projection operator along the $z$-axis is given simply by $\left\langle S_{z}\right\rangle=\operatorname{Tr}\left[\rho(t) S_{z}\right]$, which is written in closed form as follows:

$$
\left\langle S_{z}\right\rangle=\frac{4 \eta \operatorname{Im}(J)+(1-2 \gamma)\left(|I|^{2}-|K|^{2}\right)}{1+2 \eta \operatorname{Re}(I K)+(1-\gamma)\left(|I|^{2}+|K|^{2}\right)+2 \gamma|J|^{2}}
$$

We illustrate the calculation with an example for sinusoidal applied fields of the form $B_{0}(t)=B_{0} \sin \left(\omega_{0} t\right)$ and $b(t)=$ $b e^{i \omega t}$. In Fig. 1, we compare the exact numeric solution with equation (25), for the following values of parameters: $E / D=0.0075, k_{B} T / D=0.01$, with $\omega_{0} / D=0.05, \omega / D=1$, and $\mu B_{0} / D=1, \mu b / D=0.005$. Notice the good agreement between exact and approximate results. Even oscillations at long times, are accurately described by our modified perturbation scheme. Next, we compare with the usual Kubo linear response theory, where $\mathcal{H}_{<}$(see relation (12)) is considered as the unperturbed Hamiltonian. The calculation is standard and yields

$$
\left\langle S_{z}(t)\right\rangle_{K u b o}=-4 \mu \eta \int_{0}^{t} d t^{\prime} B_{0}\left(t^{\prime}\right) \sin \left[4 E\left(t-t^{\prime}\right)\right] .
$$

Note, that in first order, $\left\langle S_{z}(t)\right\rangle_{K u b o}$ is linear in $B_{0}\left(t^{\prime}\right)$ and does not depend on the transverse component $b(t)$. On the other hand, contributions of higher order in $E$ are included, as expected from the choice of the unperturbed Hamiltonian. Relation (26) can be written in closed form, for an oscillating field of the form

$$
B_{0}(t)=\left\{\begin{array}{l}
B_{o} \sin \left(\omega_{0} t\right), \text { for } t \geq 0 \\
0, \text { for } t<0
\end{array}\right.
$$

yielding

$$
\left\langle S_{z}(t)\right\rangle_{\text {Kubo }}=-4 \eta \mu B_{o}\left[\frac{\omega_{0} \sin (4 E t)-4 E \sin \left(\omega_{0} t\right)}{\omega_{0}^{2}-16 E^{2}}\right]
$$




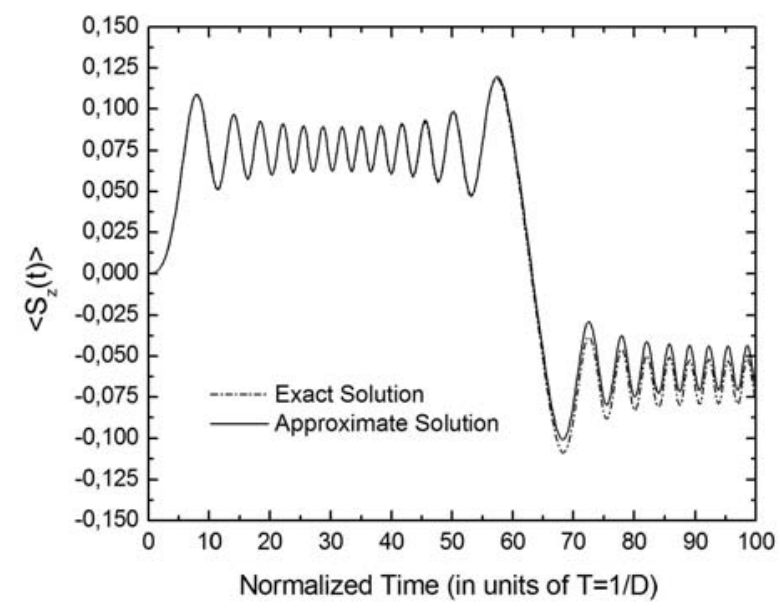

FIG. 1: Dynamics of the thermal average of the spin operator $S_{z}$, calculated by exact numeric methods (dash-dotted line) and through the modified interaction picture, after (25)(solid line). Values of parameters are $E / D=0.0075, k_{B} T / D=0.01, \omega_{0} / D=0.05, \omega / D=1$, $\mu B_{0} / D=1$, and $\mu b / D=0.005$. For explanation of the different symbols, see the text.

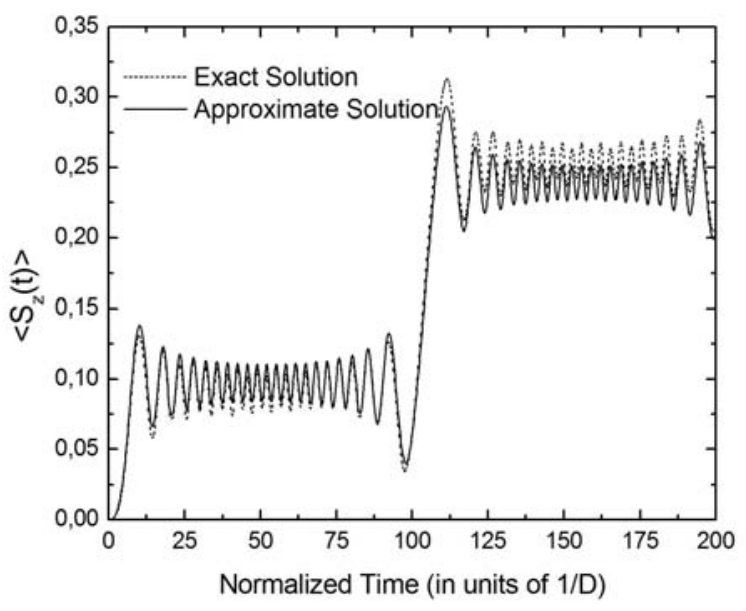

FIG. 2: Dynamics of the thermal average of the spin operator $S_{z}$ in the resonant case, $\omega_{0}=4 E$, calculated by exact numeric methods (dashed line) and through the modified interaction picture, after (25)(solid line). Values of parameters are $E / D=0.0075, k_{B} T / D=0.01, \omega_{0}=4 E$, $\omega / D=1, \mu B_{0} / D=1$, and $\mu b / D=0.015$. For explanation of the different symbols, see the text.

Kubo's formula has several shortcomings: in addition of not including the contribution of the transverse applied field in first order, the resonant denominator in (27) may produce divergent responses. By contrast, a calculation of the time response for the resonant case, $\omega_{0}=4 E$ using the modified version of perturbation theory gives a very satisfactory result. The resonant behavior is illustrated in Fig. 2, where we compare the exact numerical solution with equation (25) using $B_{0}(t)=B_{0} \sin \left(\omega_{0} t\right), b(t)=b e^{i \omega t}$ and the following parameter values: $E / D=0.0075, k_{B} T / D=0.01$, with $\omega_{0}=4 E$, $\omega / D=1$, and $\mu B_{0} / D=1, \mu b / D=0.015$. Away from the resonant condition, both perturbation schemes coincide, if in relation (25) we neglect contributions of $b(t)$ and get the linear term in $B_{0}(t)$. In turn, relation (27) has to be expanded linearly in $E$, for small times. The result is

$$
\left\langle S_{z}\right\rangle \approx-16 \eta \mu B_{o} E\left(\frac{\omega_{0} t-\sin \left(\omega_{0} t\right)}{\omega_{0}^{2}}\right) .
$$

\section{CONCLUSION}

In summary, in this manuscript we have demonstrated the possibility to go beyond the Kubo linear response theory through the use of a modified version of the interaction picture, suitable for problems in which a non-perturbed time-dependent Hamiltonian $\mathcal{H}_{0}(t)$ can be diagonalized at all times by the same basis. In such a case, the results of our perturbation method agree very well with the exact solution, even to first order and for very long times. The spin 
dynamics of molecular magnets was studied using the density matrix formalism to obtain the evolution of the magnetization. This is an important parameter, which can be measured experimentally. If large magnetic molecules are used to store quantum information, one has to keep track of the time-evolution of the states initially prepared. In this paper, we have probed our method to study the quantum dynamics of a simple molecular magnet of $\operatorname{spin} S=1$. Calculations for high-spin molecular magnets are in progress.

\section{Acknowledgements}

C. A. D. and G. G. C. would like to acknowledge partial financial support from CNPq (Conselho Nacional de Desenvolvimento Científico e Tecnológico, Brazil) through the projects 555517/2006-3 (Edital MCT/CNPq 42/2006) and $304382 / 2005-1$, respectively.
[1] MRS Bulletin, Molecule-Based Magnets, November 2000 , Vol. 25, issue 11 .

[2] J. R. Friedman, M. P. Sarachik, J. Tejada and R. Ziolo, Phys. Rev. Lett. 76 (1996), 3830.

[3] C.A. Dartora, G.G. Cabrera and K.Z. Nobrega, Brazilian Journal of Physics 37 (2007), 1147.

[4] R. Sessoli, D. Gatteschi and M.A. Novak, Nature 365 (1993), 141.

[5] L. D. Landau, Phys. Z. Sowjetunion 2 (1932), 46.

[6] C. Zener, Proc. R. Soc. London, Ser. A 137 (1932), 696.

[7] E.C.G. Stueckelberg, Helv. Phys. Acta 5 (1932), 369.

[8] H. Nakamura, in Dynamics of Molecules and Chemical Reactions, edited by R. E. Wyatt and J. Z. H. Zhang (Marcel Dekker, New York, 1996).

[9] F. B. Anders and A. Schiller, Phys. Rev. Lett. 95, 196801
(2005); S. R.White and A. E. Feiguin, Phys. Rev. Lett. 93, 076401 (2004).

[10] A. B. Migdal and V. P. Krainov, Approximation Methods in Quantum Mechanics (Benjamin, New York, 1969).

[11] J. J. Sakurai, Modern Quantum Mechanics (Revised Edition) (Addison-Wesley, Reading, 1994).

[12] C. Calero, E.M. Chudnovsky, and D.A. Garanin, Phys. Rev. B 72 (2005), 024409;

[13] W. Wernsdorfer, S. Bhaduri, C. Boskovic, G. Christou and D. N. Hendrickson, Phys. Rev. B 65 (2002), 180403.

[14] S. Miyashita, K. Saito, H. Kobayashi, and H. de Raedt, RIKEN Review 27 (2000), 57.

[15] M. Abramowitz and I.A. Stegun, editors, Handbook of Mathematical Functions (Dover, New York, 1972). 